\title{
Securing Mesh with Delayed Absorbable Suture Does Not Increase Risk of Prolapse Recurrence Following Robotic Sacral Colpopexy
}

Authors: CR Powell, Isamu Tachibana, Bridget Eckrich, Jeffrey Rothenberg, and Jon Hathaway

Introduction and Objective: NON-absorbable monofilament suture is traditionally used to secure vaginal mesh for Robotic Assisted Sacral Colpopexy (RASC) but can become exposed postoperatively requiring invasive vaginal removal. Polydioxanone delayed absorbable suture may avoid this. We sought to determine the objective and subjective impact of suture choice for mesh fixation.

Methods: A cohort study was undertaken using a prospective registry and subjects were grouped based on type of suture at the time of RASC. Apical failure was defined as C point descent of greater than $2 \mathrm{~cm}$, Anterior compartment failure was defined as POP-Q Ba point of $>0$, and Posterior compartment failure was defined as Bp point of $>0$. Patient reported outcomes included UDI-6 and QoL. 2-tail T-test and Chi-squared were used for analysis.

Results: 119 women underwent RASC between 2009 and 2016. Patients had similar preoperative characteristics (Table 1). All POP-Q, UDI-6, and QoL scores improved postoperatively (Table 1 and 2). Apical failure was noted in 0 , Anterior failure was noted in 7 (average $\mathrm{Ba}+1.1 \mathrm{~cm}$ in failures), and Posterior failure was noted in 4 (mean Bp $+1.0 \mathrm{~cm}$ ) at 16 months' follow up. Failures in the anterior compartment were much more common in the NON-absorbable monofilament cohort (Table 2). Failures in the apical and posterior compartments were not significantly different between groups. 9 suture erosions were noted in the NON-absorbable monofilament cohort, 5 requiring excision in the clinic and 2 in the operating room. 2 suture erosions were noted in the delayed-absorbable cohort, 0 required excision. Postoperative UDI-6 and QoL scores did not vary significantly between groups ( $5.3 \pm 4.0$ vs. $5.1 \pm 4.0, p=N S, 2.8 \pm 2.0$ vs. $2.8 \pm 2.2, p=N S)$.

Conclusion: Securing mesh with delayed-absorbable monofilament did not appear to increase risk of failure in patients undergoing RASC and eliminates the need for suture excision postoperatively. 
Introduction:

Abdominal sacral colpopexy has been the most durable treatment for severe pelvic organ prolapse (POP). Traditionally this has required an open incision, and this likely dissuaded many women from electing for surgery, because of perceived surgical morbidities ${ }^{1}$. Laparoscopic sacral colpopexy has been noted to have similar outcomes, with shorter hospital stays and improved pain, but has not been widely adopted by surgeons specializing in $\mathrm{POP}^{2}$. After the introduction of the robotic da Vinci Surgical platform (Intuitive Surgical, Sunnyvale, CA, USA) to manage POP, utilization of robotic assisted laparoscopic sacral colpopexy has been increasing nationwide ${ }^{3}$. Recent meta-analysis demonstrated equivalent outcomes for abdominal sacral colpopexy and robotic-assisted sacral colpopexy (RASC). The authors reviewed over 1400 RASC procedures described in the literature highlighting the growing popularity of this approach ${ }^{4}$. The complications of RASC are not as well described in the literature as those for the open technique. Mesh extrusion has received significant media and scientific attention recently and is well recognized by most patients. NON-absorbable monofilament polypropylene suture (Prolene ${ }^{\circledR}$, Johnson\&Johnson, New Brunswick, NJ, USA) is typically used to secure the mesh, and suture extrusion can be very distressing to the patient and often requires invasive suture trimming in the clinic or operating room. Some have suggested anchoring mesh to the vaginal wall using monofilament delayed absorbable polydioxanone suture

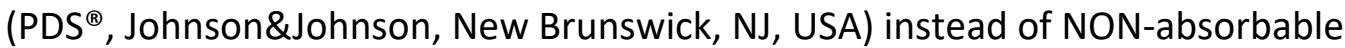
monofilament to avoid this complication. Authors' note: the use of the trade name is not intended to imply that the branded product is superior, but it was thought that these trade names would be familiar to many of the readers and so are used but sparingly. In addition, some time may be saved by placing these sutures in a running fashion to secure mesh as opposed to the traditionally described interrupted technique. The aim of this study is to compare two techniques to secure vaginal mesh-interrupted NON-absorbable monofilament sutures versus running DELAYED-ABSORBABLE MONOFILAMENT sutures. Data is taken from a single-institution experience with RASC and is retrospective. The hypothesis is that both techniques will deliver similar outcomes, but the latter will avoid the need to extract sutures that perforate the vaginal wall. 
Materials and Methods:

After institutional review board approval, a systematic review of the prospectively collected Indiana University Sacral colpopexy database was undertaken to identify women who underwent robotic-assisted sacral colpopexy (RASC) between August 2009 and December 2016. The results of the last documented clinic follow up were reported. All patients undergoing simultaneous hysterectomy had gynecologic consultation. Technique for hysterectomy (robotic supracervical hysterectomy versus robotic total hysterectomy) was left to the discretion of the consulting gynecologist (JMR and JH). Demographic variables were collected from the pre-operative, intra-operative and post-operative periods.

Concomitant procedures, such as mid urethral sling, culdoplasty, or vaginal cystocele repair were performed in some, but not all, patients. The decision to perform concomitant procedures was left to the discretion of the urologist and patient (CRP). All patients were counseled about the possibility of postoperative urinary incontinence, suture extrusion, mesh erosion, and the possible need for a staged continence procedure during preoperative consultations.

RASC was performed using da Vinci ${ }^{\circledR}$ Surgical System (Intuitive Surgical, Sunnyvale, CA, USA). The technique can be broken into 11 stages and viewed on the Journal of Endourology Video Atlas (http://online.liebertpub.com/loi/END). Special attention should be paid to a few areas:

I. Positioning and port placement: The patient is positioned on the table as shown in Figure 1, it is important that the arms are tucked to the sides and padded generously. Take care to pad between IV catheters and the skin. The four-arm robotic port site location on the abdomen is detailed in Figure 2. The robot can be positioned between the legs, or to the side of the left leg to maintain access to the vaginal area (aka "side docking"). The side-docking technique is may lead to crashing of arm 2 and 3 with the DaVinci SI ${ }^{\circledR}$ system but careful planning can avoid this. An assistant port can be $5 \mathrm{~mm}$ which is not large enough to pass needles, $8 \mathrm{~mm}$, or $12 \mathrm{~mm}$ depending on the surgeon's preference. After placing ports, 
twenty-seven degrees of Trendelenburg position is used to allow the bowel to fall out of the pelvis. Initially, vaginal retraction was performed with a hand-held Lucite vaginal retractor or sponge-stick. A bed-mounted vaginal manipulator is also available (Uterine Positioning System, Cooper Surgical, Trumbull, CT, USA) to facilitate anterior and posterior dissection of the vagina and is more stable for vaginal wall suturing. This is shown in Figures 3 and 4.

II. Anterior and Posterior vaginal wall dissection: The bladder can be filled with saline to better identify the reflection of the bladder. The anterior dissection is taken down to the level of bladder trigone $(5-10 \mathrm{~cm})$ while the posterior vaginal wall dissection is carried to the rectovaginal space $(5-10 \mathrm{~cm})$. A useful measurement tool is the gray insulation on the $\mathrm{DaVinci}{ }^{\circledR}$ monopolar scissor device which measures $5 \mathrm{~cm}$ from the tip of the scissor to the proximal end of the insulation.

III. Secure mesh to the anterior and posterior vaginal wall: This can be done in interrupted or running fashion, with delayed absorbable suture or NON-absorbable suture. The results section will describe our results with both techniques. The key is to use the \#3 robotic arm and intra-vaginal retractor to optimize exposure. In addition to polypropylene mesh, porcine dermis and autologous fascia lata can be used.

Pelvic Organ Prolapse Quantification (POP-Q) exam was performed and recorded preoperatively and post-operatively on every patient. Treatment failure was defined as recurrent apical prolapse greater than $2 \mathrm{~cm}$ or any leading edge beyond the hymen $(\mathrm{Ba}>0$ or $\mathrm{Bp}>0)$. Surgical characteristics examined included type of sutures used for mesh fixation, prior surgical history including prolapse and incontinence procedures, POP-Q exam, and validated quality of life instruments such as UDI-6 and IIQ-7. Perioperative complications included vaginal suture extrusion, vaginal mesh extrusion, port site hernia, deep venous thrombus (DVT), atrial fibrillation, mesh extrusion, prolonged intubation, and wound infection. The decision to remove sutures was made by the patient and the surgen, and the decision to remove them in the operating room was made by the patient. The study was approved by the Indiana University School of Medicine Institutional Review Board. Statistical analyses were performed using 
Microsoft Excel ${ }^{\circledR}$ (Microsoft Corp, Redmond, Washington, USA) and SPSS version 20.0 (IBM, Chicago, IL, USA). Categorical variables were analyzed for significance with ChiSquared analysis and continuous variables were analyzed with 2-tailed Student's T-test.

\section{Results:}

119 women underwent RSC between 2009-2016. Patients had similar preoperative characteristics (Table 1). Fifty-three presented with hysterectomy prior to RSC, 15 with prior vaginal prolapse repair, as well as 8 with prior incontinence procedures. Patients also underwent procedures at the time of colpopexy. 66 underwent robotic supracervical or total hysterectomy, 34 had mid urethral sling at the time of colpopexy, 22 had culdoplasty for enterocele, and 5 had concomitant anterior vaginal wall suspension. POPQ, UDI-6, and QoL scores improved post operatively (Table 2). Apical failure was not noted in any, Anterior failure was seen in 7 (average Ba was $+1.1 \mathrm{~cm}$ in failures), and Posterior failure was seen in 4 (mean Bp was $+1.0 \mathrm{~cm}$ ) at 16 months' mean follow up. Failures in the anterior compartment were much more common in the NON-absorbable monofilament cohort (Table 3). Failures in the apical and posterior compartments were not significantly different between groups. 9/49 suture erosions were noted in the NONabsorbable monofilament cohort, 5 requiring excision in the clinic, 2 in the operating room, and 2 not requiring intervention. 2/70 suture erosions were noted in the DELAYEDABSORBABLE MONOFILAMENT cohort, 0 required intervention. Post-operative UDI-6 and QoL scores did not vary significantly between groups ( $5.3 \pm 4.0$ vs. $5.1 \pm 4.0, p=N S, 2.8 \pm 2.0$ vs. $2.8 \pm 2.2, p=N S)$. Complications did occur. 2 wound infections were managed with antibiotics (Clavien-Dindo classification Grade $\mathrm{II}^{7}$ ) and 2 vaginal mesh erosions were noted. One prolonged intubation occurred, and she was successfully extubated on POD\#2. One new-onset episode of atrial fibrillation was observed. Three port site hernias were noted post operatively, always at the $12 \mathrm{~mm}$ lateral assistant port site, one required operative intervention (Clavien-Dindo Grade IIIb), and the other two were managed nonoperatively (Clavien-Dindo Grade I). One of the vaginal mesh erosion events occurred following total hysterectomy at the time of colpopexy, requiring surgical intervention (Clavien-Dindo Grade IIIb). The other patient had undergone hysterectomy prior to RASC, 
had no recognized vaginotomy, was asymptomatic, and chose non-operative management (Clavien I).

\section{Discussion:}

Compared to other contemporary RASC series, this series is the first to compare failure rates between delayed absorbable monofilament suture (PDS ${ }^{\circledR}$ ) and permanent NONabsorbable monofilament (Prolene ${ }^{\circledR}$ ). Vaginal suture extrusion is not reported as a complication in many series, but certainly contributes to patient morbidity, and appears to be more common than vaginal mesh exposure, at least in this series. The low rates of suture extrusion made descriptive statistics less meaningful, but the trend in this series is clear. Ploumidis et al. reported an intraoperative and postoperative complication rate of $3.2 \%$ in a cohort of 95 women who underwent RASC ${ }^{8}$. In that study, less than half of the patients had prior abdominal surgery and no patients had concomitant hysterectomy. In one large RASC series, Nosti et al. found an overall complication rate of $7 \%$ in a group of 262 women of which approximately $43 \%$ of women had prior abdominal surgeries ${ }^{9}$.

Women in the present series were more likely to have previous hysterectomy (44.6\%) and concomitant hysterectomy (55.4\%).

Our port site hernia rate of $2.5 \%$ was higher than the reported rates of 0 to $1.6 \%$ in other contemporary series $^{9-11}$, but not all RASC series report on port site hernia as a complication.

The mesh extrusion rate in the current study is comparable to the rates reported by a recent meta-analysis of RASC $(1.7 \% \text { vs. } 0-19 \%)^{4}$. The single patient in the present series who required operative repair of her exposed mesh had undergone concomitant total hysterectomy. Previous studies have shown that concomitant total hysterectomy is associated with a high incidence of mesh extrusion ranging from $16 \%$ to $23 \%$ after sacrocolpopexy ${ }^{12,13}$. Because of this analysis we now counsel the patients requiring hysterectomy to undergo supracervical hysterectomy if there is no increases risk for cervical cancer, and since that time, no additional mesh exposure events following supracervical hysterectomy were noted in this series. In a recent study of 277 women undergoing abdominal sacral colpopexy (not RASC) with either a concomitant 
supracervical hysterectomy or no hysterectomy because it had been done previously, the authors found no difference in mesh extrusion in the supracervical group $(2 / 195=0.5 \%$, compared to $2 / 82=2.4 \%$ ) in the total hysterectomy group ${ }^{14}$.

Vaginal suture extrusion in our study is higher than another laparoscopic series $(9.2 \%$ vs. $6 \%)^{15}$. Most patients with suture extrusions presented with no symptoms, only noted by the examining urologist. However, some may present with vaginal discharge or dyspareunia. Vaginal suture extrusion is likely a technical error from placement of NONabsorbable sutures through full thickness of vagina. In an effort to minimize suture extrusions, we switched to delayed absorbable polydioxanone sutures for mesh fixation. A main concern with using absorbable sutures is the durability of mesh fixation. Porcine models demonstrate that majority of the final strength of tissue in-growth into polypropylene mesh is achieved by 12 weeks postoperatively, and that 2 weeks yields significantly lower bond of mesh to fascia ${ }^{16}$. Polydioxanone suture loses only $50 \%$ of its tensile strength by 6 weeks, whereas Maxon ${ }^{\circledR}$ (polyglyconate) loses $50 \%$ of its strength by 3 weeks and Vicryl ${ }^{\circledR}$ by 2 weeks ${ }^{17}$. The rate of recurrent prolapse was not influenced by the type of mesh fixation suture used in our study. A recent study by Borahay et al. also found that delayed absorbable sutures is effective in RASC patients with no apical prolapse at a median follow up of 17 months ${ }^{18}$. A randomized controlled trial with longer followup is needed to address better address this issue.

There are several limitations in this study including those common to retrospective reviews. The data came from a single institution, single surgeon, and the sample size is small relative to the number of suture extrusion events. A trial to truly determine the durability of delayed absorbable sutures would need to be randomized and blinded, but the present work suggests results are equivalent.

\section{Conclusions:}

Securing mesh with DELAYED-ABSORBABLE MONOFILAMENT does not appear to increase risk of failure in patients undergoing RASC and eliminates the need for suture excision post operatively. The technique for RASC described above yields durable outcomes equal to that in the contemporary literature. This work justifies more study into the use of delayed 
absorbable sutures to secure polypropylene mesh during RASC, and more study is needed before patients can be counseled that delayed absorbable suture can match permanent suture for durability. 
REFERENCE

1. Nygaard IE, McCreery R, Brubaker L, et al. Abdominal sacrocolpopexy: a comprehensive review. Obstet Gynecol. 2004 Oct;104(4):805-23. PubMed PMID: 15458906. Epub 2004/10/02. eng.

2. Ganatra AM, Rozet F, Sanchez-Salas R, et al. The current status of laparoscopic sacrocolpopexy: a review. Eur Urol. 2009 May;55(5):1089-103. PubMed PMID: 19201521. Epub 2009/02/10. eng.

3. Flack CK, Monn MF, Patel NB, Gardner TA, Powell CR. National Trends in the Performance of Robot-Assisted Sacral Colpopexy. J Endourol. 2015 Jul;29(7):777-83. PubMed PMID: 25654587.

4. Serati M, Bogani G, Sorice P, et al. Robot-assisted Sacrocolpopexy for Pelvic Organ Prolapse: A Systematic Review and Meta-analysis of Comparative Studies. Eur Urol. 2014 Mar 6. PubMed PMID: 24631406. Epub 2014/03/19. Eng.

5. Muffly TM, Diwadkar GB, Paraiso MF. Lumbosacral osteomyelitis after robotassisted total laparoscopic hysterectomy and sacral colpopexy. Int Urogynecol J. 2010 Dec;21(12):1569-71. PubMed PMID: 20532751. Epub 2010/06/10. eng.

6. Nosseir SB, Kim YH, Lind LR, Winkler HA. Sacral osteomyelitis after robotically assisted laparoscopic sacral colpopexy. Obstet Gynecol. 2010 Aug;116 Suppl 2:513-5. PubMed PMID: 20664437. Epub 2010/07/29. eng.

7. Clavien PA, Barkun J, de Oliveira ML, et al. The Clavien-Dindo classification of surgical complications: five-year experience. Annals of surgery. 2009 Aug;250(2):187-96. PubMed PMID: 19638912. Epub 2009/07/30. eng.

8. Ploumidis A, Spinoit AF, De Naeyer G, et al. Robot-assisted sacrocolpopexy for pelvic organ prolapse: surgical technique and outcomes at a single high-volume institution. Eur Urol. 2014 Jan;65(1):138-45. PubMed PMID: 23806518. Epub 2013/06/29. eng.

9. Nosti PA, Umoh Andy $U$, Kane $S$, et al. Outcomes of abdominal and minimally invasive sacrocolpopexy: a retrospective cohort study. Female pelvic medicine \& reconstructive surgery. 2014 Jan-Feb;20(1):33-7. PubMed PMID: 24368486. Epub 2013/12/26. eng. 
10. Brubaker L, Nygaard I, Richter HE, et al. Two-year outcomes after sacrocolpopexy with and without burch to prevent stress urinary incontinence. Obstet Gynecol. 2008 Jul;112(1):49-55. PubMed PMID: 18591307. Pubmed Central PMCID: 2614233. Epub 2008/07/02. eng.

11. Culligan PJ, Gurshumov E, Lewis $\mathrm{C}$, et al. Subjective and objective results 1 year after robotic sacrocolpopexy using a lightweight Y-mesh. Int Urogynecol J. 2014 Jun;25(6):731-5. PubMed PMID: 24264283. Epub 2013/11/23. eng.

12. Siddiqui NY, Geller EJ, Visco AG. Symptomatic and anatomic 1-year outcomes after robotic and abdominal sacrocolpopexy. Am J Obstet Gynecol. 2012 May;206(5):435 e1-5. PubMed PMID: 22397900. Epub 2012/03/09. eng.

13. Stepanian AA, Miklos JR, Moore RD, Mattox TF. Risk of mesh extrusion and other mesh-related complications after laparoscopic sacral colpopexy with or without concurrent laparoscopic-assisted vaginal hysterectomy: experience of 402 patients. J Minim Invasive Gynecol. 2008 Mar-Apr;15(2):188-96. PubMed PMID: 18312989. Epub 2008/03/04. eng.

14. Ginath S, Garely AD, Condrea A, Vardy MD. Mesh erosion following abdominal sacral colpopexy in the absence and presence of the cervical stump. Int Urogynecol J. 2013 Jan;24(1):113-8. PubMed PMID: 22717784. Epub 2012/06/22. eng.

15. Cundiff GW, Varner E, Visco AG, et al. Risk factors for mesh/suture erosion following sacral colpopexy. Am J Obstet Gynecol. 2008 Dec;199(6):688 e1-5. PubMed PMID: 18976976. Epub 2008/11/04. eng.

16. Majercik S, Tsikitis V, lannitti DA. Strength of tissue attachment to mesh after ventral hernia repair with synthetic composite mesh in a porcine model. Surgical endoscopy. 2006 Nov;20(11):1671-4. PubMed PMID: 17001442.

17. Bourne RB, Bitar H, Andreae PR, Martin LM, Finlay JB, Marquis F. In-vivo comparison of four absorbable sutures: Vicryl, Dexon Plus, Maxon and PDS. Can J Surg. 1988 Jan;31(1):43-5. PubMed PMID: 2827875.

18. Borahay MA, Oge T, Walsh TM, Patel PR, Rodriguez AM, Kilic GS. Outcomes of robotic sacrocolpopexy using barbed delayed absorbable sutures. J Minim Invasive Gynecol. 2014 May-Jun;21(3):412-6. PubMed PMID: 24263027. 
Abbreviations:

Robotic Assisted Sacral Colpopexy (RASC)

Polydiaxone delayed absorbable suture (PDS ${ }^{\circledR}$ )

pelvic organ prolapse (POP)

Pelvic Organ Prolapse Quantification (POP-Q)

deep venous thrombus (DVT) 
Table 1

Table 1 Preop Demographics

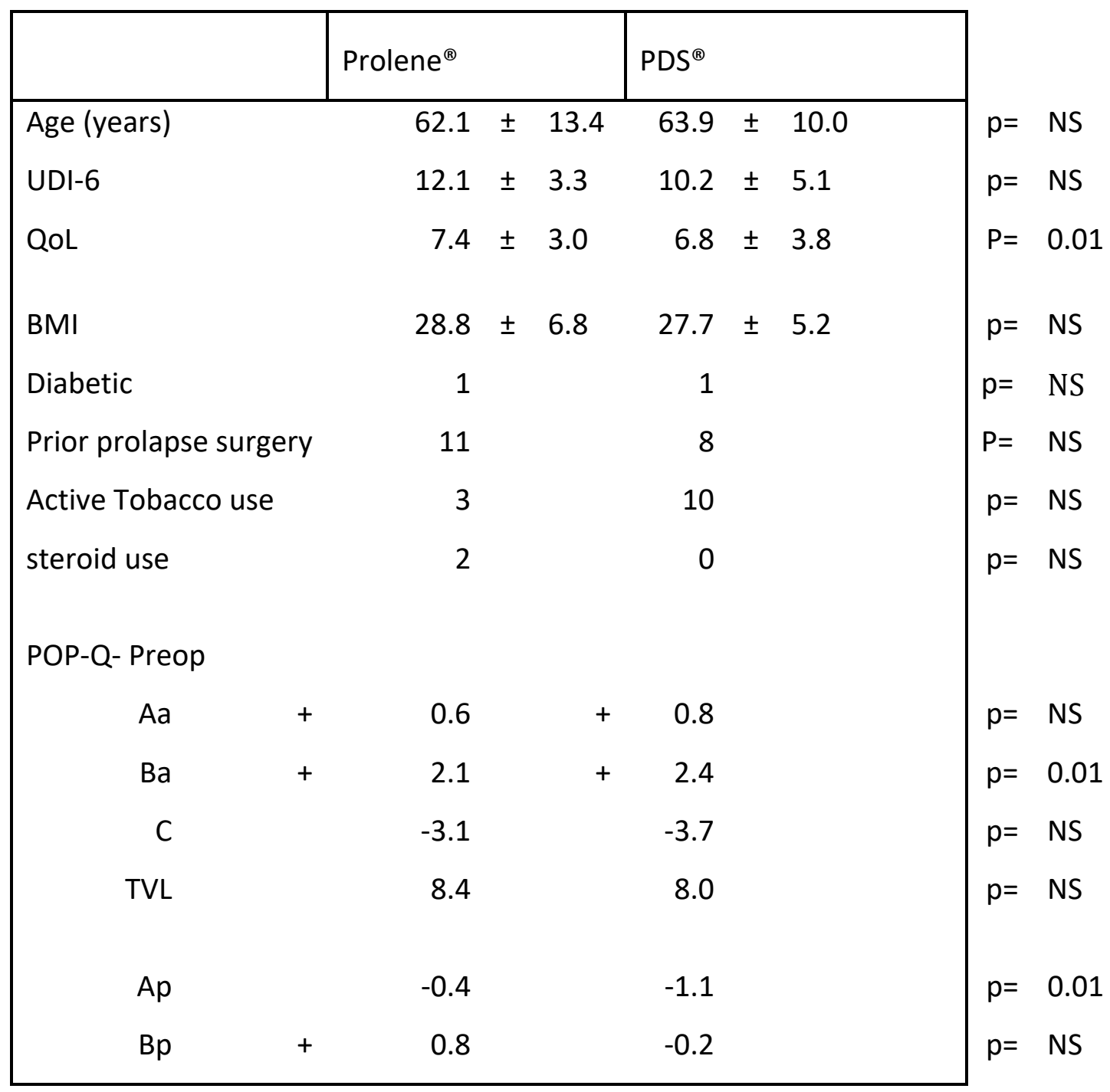


Table 2 Outcomes for Sacral Colpopexy by suture type

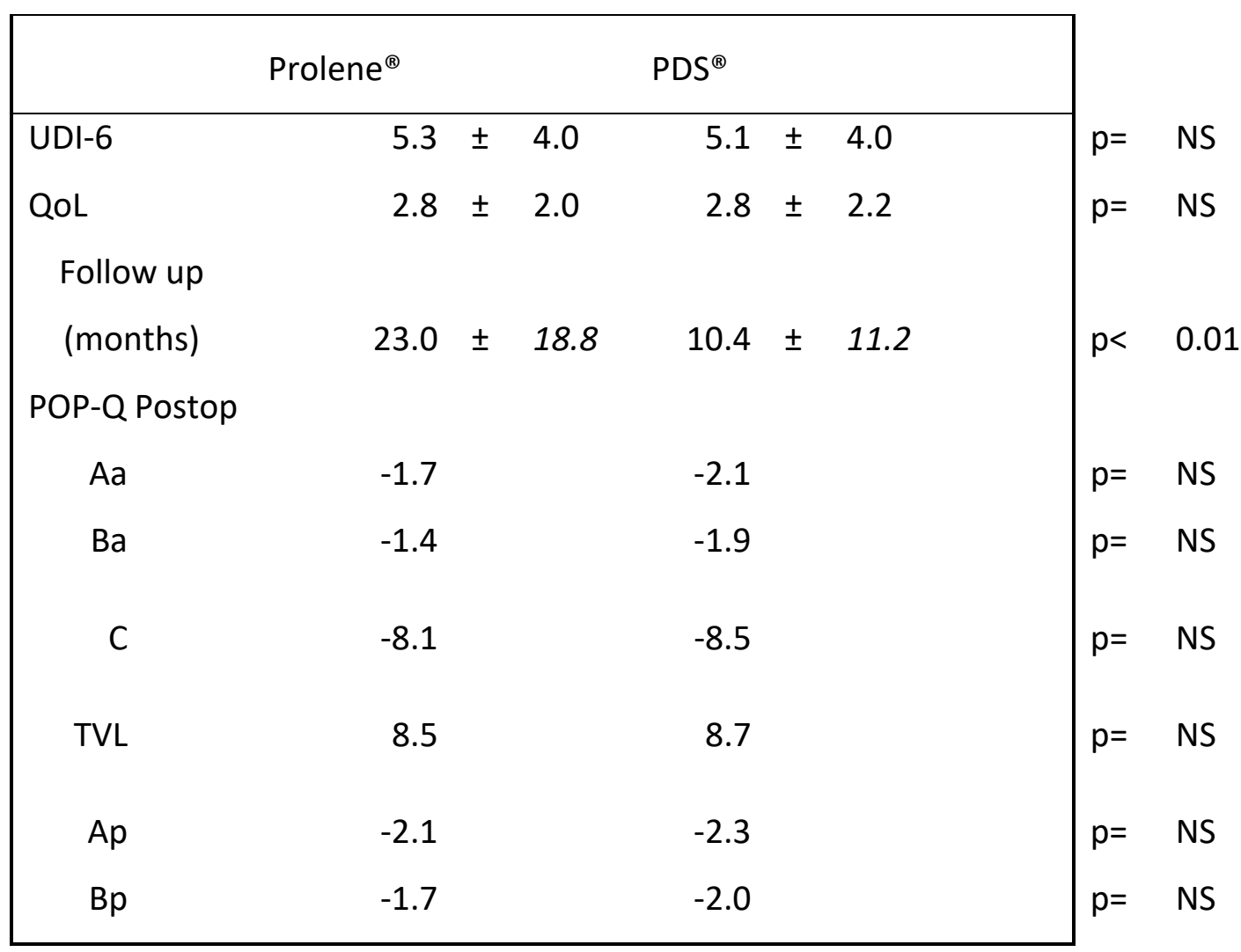


Table 3. Failure of repair by compartment and type of suture used to secure mesh. Suture extrusion by suture type. Significance is by Chi-Squared analysis.

\begin{tabular}{|c|c|c|c|}
\hline REPAIR FAILURE & Prolene $^{\circledR}$ & $\mathrm{PDS}^{\circledR}$ & \\
\hline \multirow{3}{*}{$\begin{array}{l}\text { at ANTERIOR compartment } \\
\text { at POSTERIOR } \\
\text { compartment }\end{array}$} & $0 / 49$ & $0 / 70$ & $p=N S$ \\
\hline & $6 / 49$ & $1 / 70$ & $p=0.012$ \\
\hline & $3 / 49$ & $1 / 70$ & \multirow[t]{4}{*}{$p=N S$} \\
\hline & & excision & \\
\hline Prolene ${ }^{\circledR}$ suture erosion & $9 / 49$ & $7 / 9$ & \\
\hline $\mathrm{PDS}^{\circledast}$ suture erosion & $2 / 70$ & $0 / 2$ & \\
\hline
\end{tabular}


Figure Legends

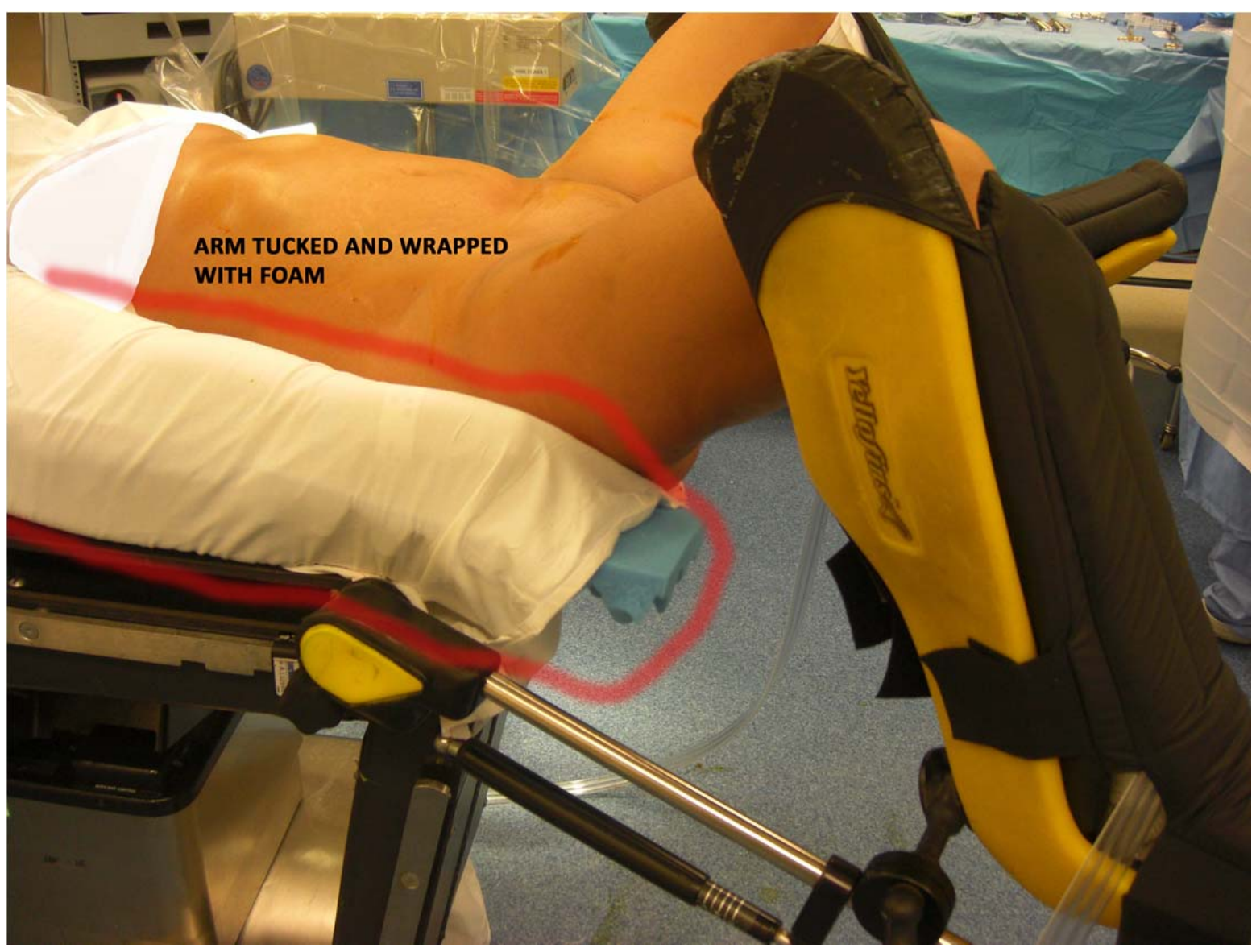

Figure 1. Arm tucked and wrapped with foam, legs in stirrups. 


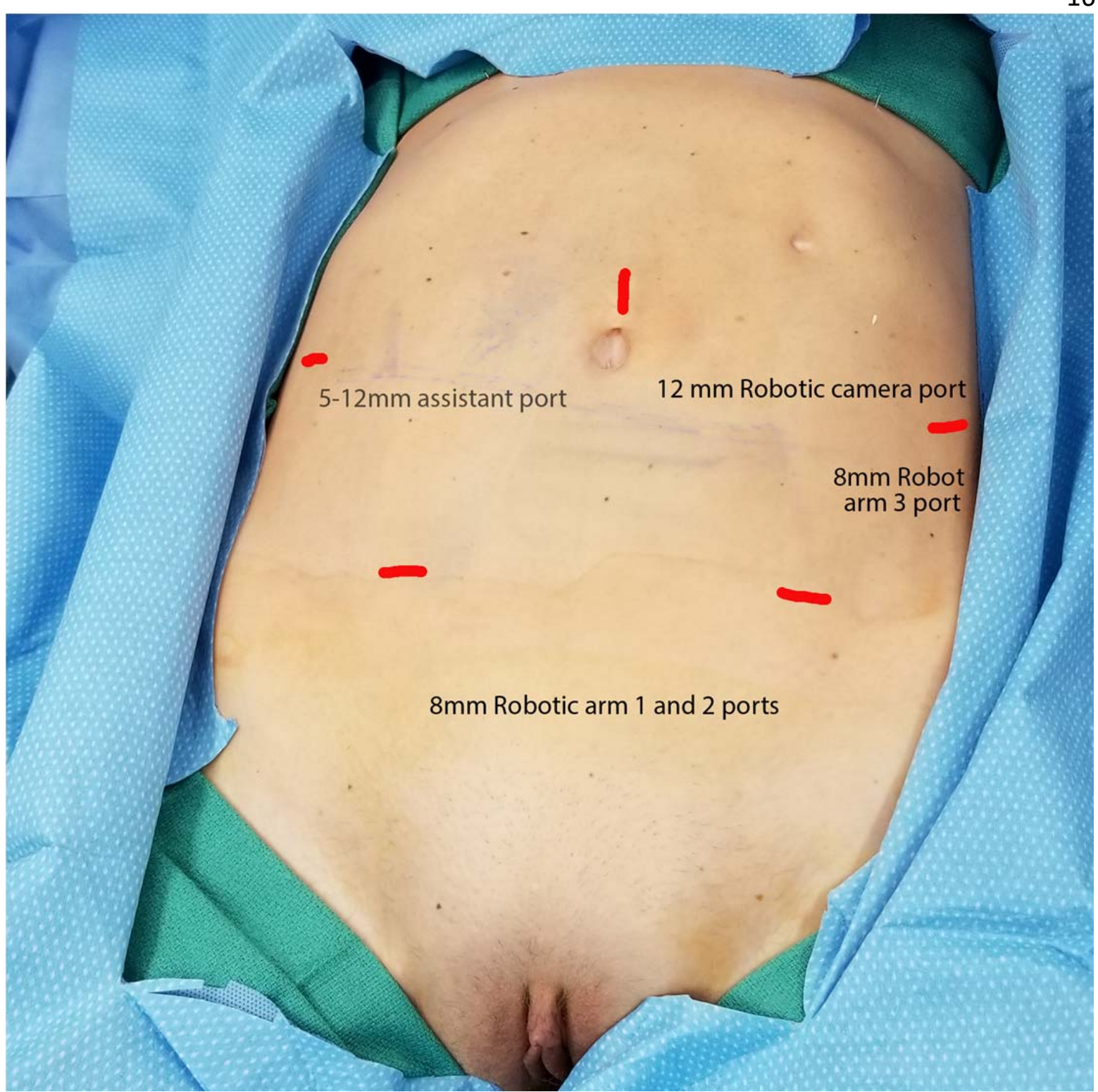

Figure 2. Port site locations on abdomen using 4-Robotic arm setup. 


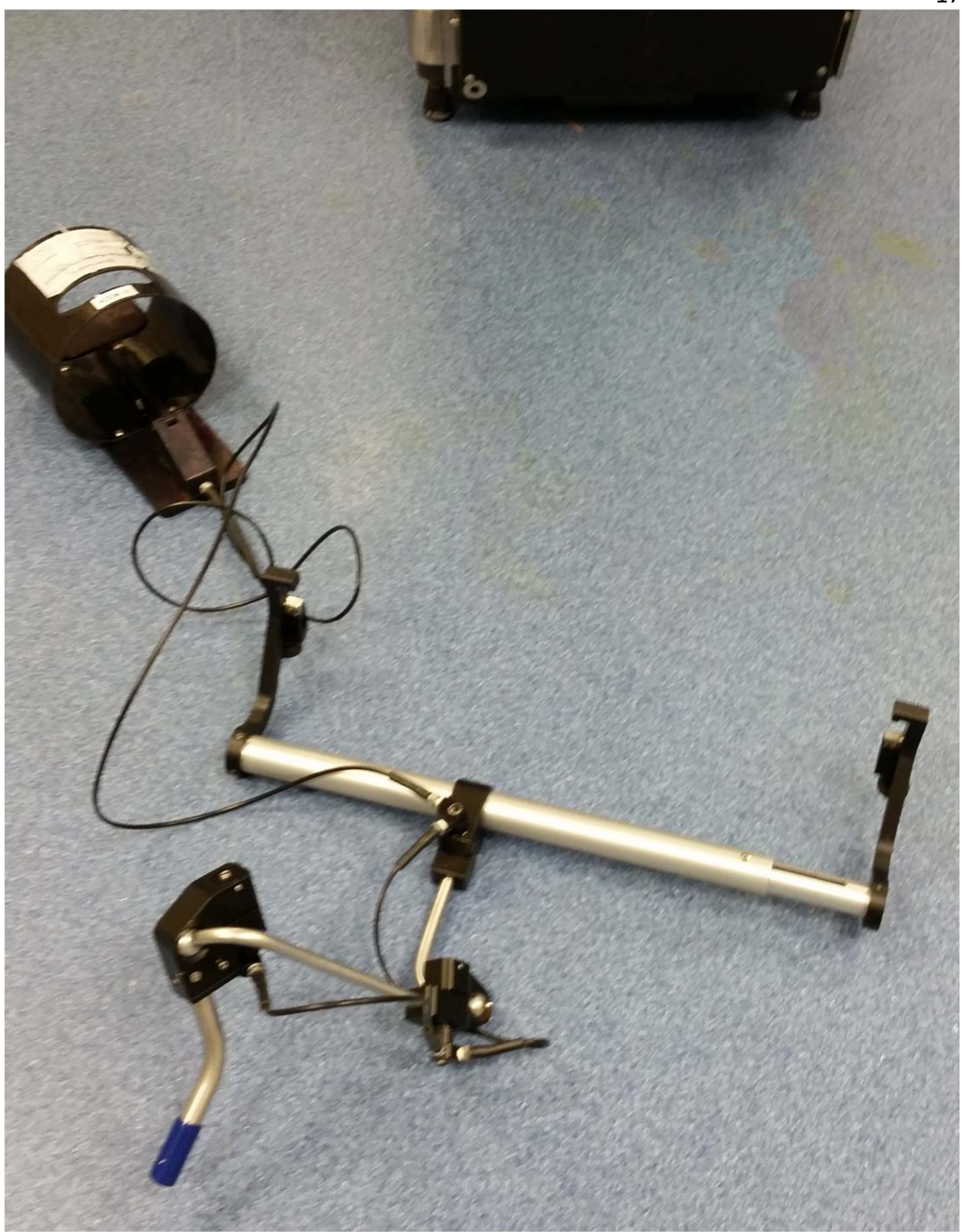

Figure 3. Bed mounted vaginal retractor prior to anchoring to bed. 
Page 18 of 18

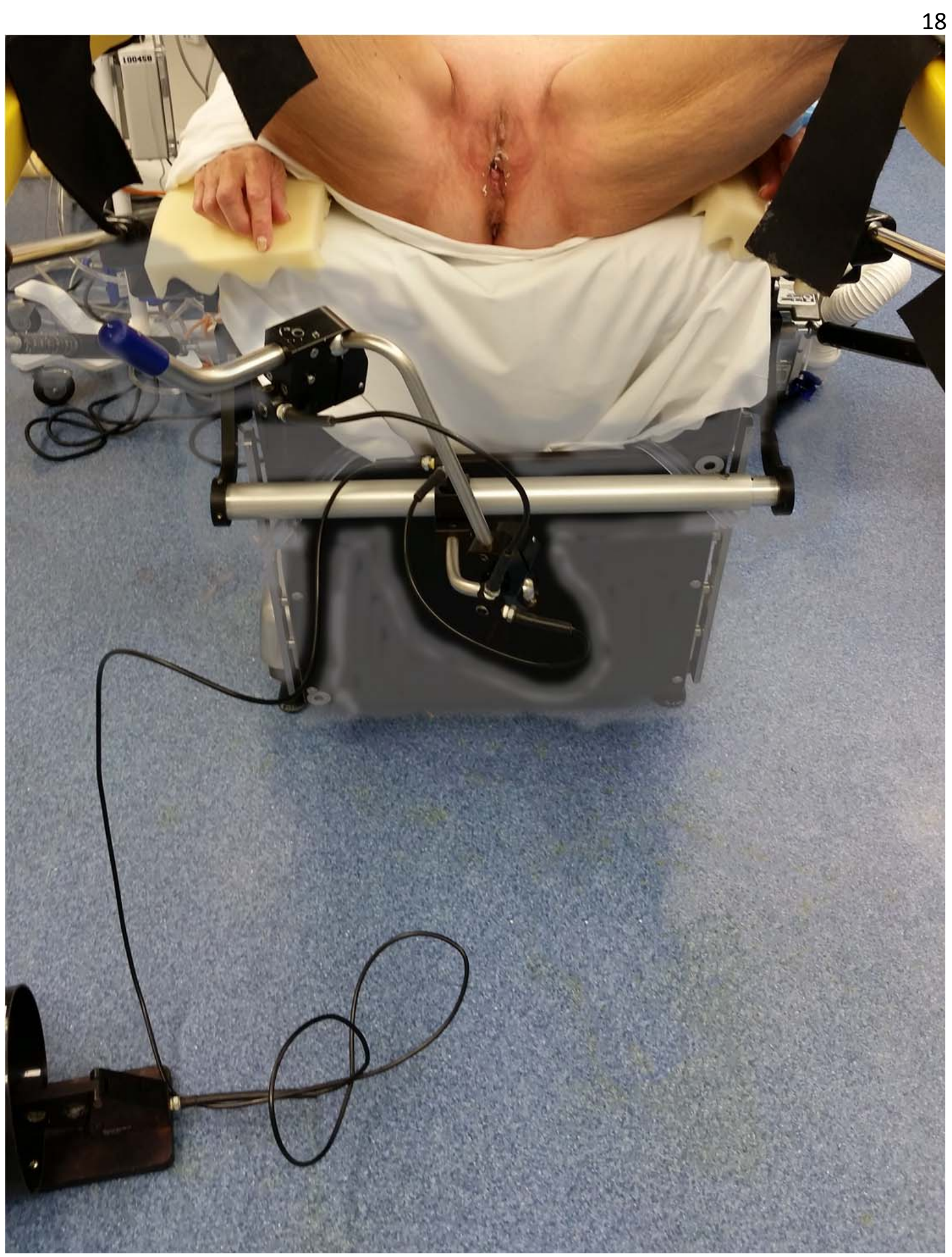

Figure 4. Bed mounted vaginal retractor after being anchored to bed. 